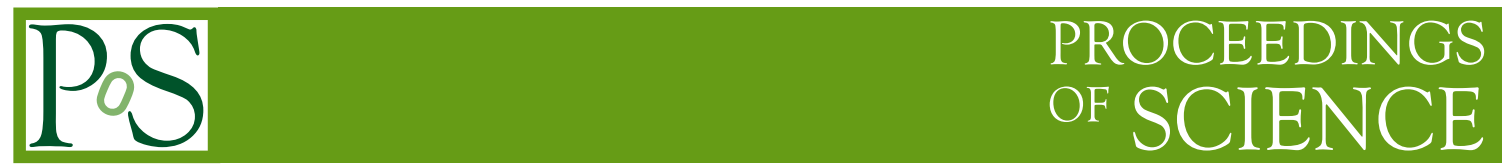

\title{
Photon-induced processes at ATLAS and CMS
}

\section{Christophe Royon*}

The University of Kansas, Lawrence, USA

E-mail: christophe.royon@ku.edu

On behalf of the ATLAS and CMS Collaborations

We present the most recent results from the ATLAS and CMS collaborations on photon-induced processes, namely the first observation of exclusive $W W$ production in $p p$ collisions and light-bylight scattering in $\mathrm{PbPb}$ collisions. The exclusive production of dileptons is also presented, and the first limit on exclusive diphoton production at high mass.

The Ninth Annual Conference on Large Hadron Collider Physics - LHCP2021

7-12 June 2021

${ }^{*}$ Speaker. 
In this short report, we will describe the most recent results on photon-induced processes from the ATLAS and CMS experiments, namely the exclusive $W W$, diphoton and dilepton production. Exclusive dilepton production is by definition a QED process, while exclusive production of two photons, as shown in Fig. 1, can be due to gluon (left) or photon-induced (right) processes. The QCD (resp. QED) production dominates at low (resp. high above $150 \mathrm{GeV}$ ) diphoton masses in $p p$ collisions. It is possible to select high diphoton or $W W$ masses in $p p$ interactions by tagging the intact protons in the final state using roman pot detectors from the CMS-TOTEM Precision Proton Spectrometer (PPS) or the ATLAS Forward Proton detectors (AFP) or by selecting exclusive events using the rapidity gap technique in the main ATLAS or CMS detectors. $\mathrm{PbPb}$ collisions allow accessing low mass photon induced processes since their cross section is enhanced by a factor $Z^{4}$ and thus dominates the QCD induced cross sections.

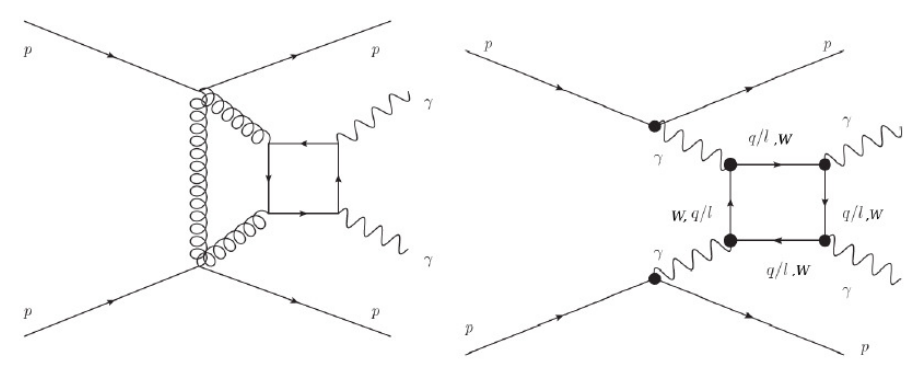

Figure 1: Exclusive diphoton production via gluon (left) and photon (right) induced processes.

\section{Observation of exclusive $W W$ production}

The ATLAS collaboration observed for the first time the Standard Model (SM) exclusive production of $W W$ bosons in photon interactions at $13 \mathrm{TeV}$ using about $139 \mathrm{fb}^{-1}$ of data [1]. Only leptonic decays of $W$ bosons ( $W W \rightarrow e v_{e} \mu v_{\mu}$ ) are considered and the exclusive selection is based on the number of tracks fitted to the primary vertex (outside $e / \mu$ ). In Fig. 2, we show the number of reconstructed tracks outside the leptons, and we clearly see an excess with respect to background at low multiplicities that leads to the first observation of exclusive $W W$ production with a cross section of $\sigma(\gamma \gamma \rightarrow W W)=3.13 \pm 0.31$ (stat.) \pm 0.28 (syst.) $\mathrm{fb}$ in the detector acceptance, in good agreement with Monte Carlo (MC) expectations.

At higher instantaneous luminosity, the search for exclusive $W W$ production becomes more difficult because of pile up and the lower efficiency of the selection method based on tracks. The solution will be to use the roman pot detectors that were installed in PPS and AFP in order to detect and measure directly the intact protons in the final state.

\section{Exclusive dilepton production}

We will now discuss the observation of exclusive dilepton production by the ATLAS and CMS collaborations in $\mathrm{PbPb}$ and $\mathrm{pp}$ collisions. The motivation to study the exclusive production 


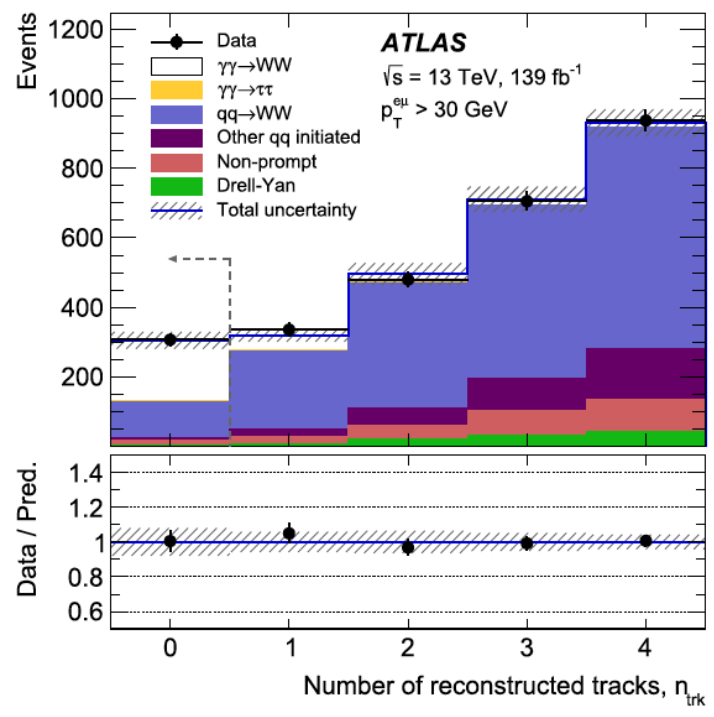

Figure 2: Number of tracks fitted to the the primary vertex (outside the leptons originating from the decays of the $W$ bosons) showing an excess at low luminosity leading to the first observation of $W W$ exclusive production by the ATLAS collaboration.

of dimuons in $\mathrm{PbPb}$ collisions is the good sensitivity to $\mathrm{Pb}$ electromagnetic form factors and to higher order effects in Coulomb interactions usually not included in MC. The CMS collaboration studied the dependence on forward neutron multiplicity as a function of $m_{\mu \mu}$ and the difference in azimuthal angle between the two muons for $p_{T, \mu}>3.5 \mathrm{GeV}$ and $8<m_{\mu \mu}<60 \mathrm{GeV},\left|y_{\mu \mu}\right|<2.4$, and differences with respect to the STARLIGHT MC [2] were observed especially when no neutron are detected in the ZDC (zero degree calorimeter) [3].

The ATLAS collaboration performed a similar study measuring muon pairs with $p_{T \mu}>4$ $\mathrm{GeV},\left|\eta_{\mu}\right|<0.4, M_{\mu \mu}>10 \mathrm{GeV}, P_{T \mu \mu}>2 \mathrm{GeV}$. The description of $0 n 0 n$ data when no neutron is detected in ZDC is described by the PYTHIA8 [4] and STARLIGHT MC including final state radiation effects, whereas the description of $X n X n$ (meaning at least one neutron detected in ZDC) requires dissociative backgrounds described using LPAIR [5, 6].

Both ATLAS and CMS collaborations also observed the quasi-exclusive production of dileptons in $p p$ collisions at high mass by tagging at least one of the two intact protons in the final state in PPS or AFP. We basically turn the LHC into a $\gamma \gamma$ collider at high luminosity and the flux of quasi-real photons is treated using the Equivalent Photon Approximation. The CMS-TOTEM collaborations measured for the first time the semi-exclusive dilepton process with one proton tag and 17 (resp. 23) events were found with protons in the PPS acceptance in the $\mu \mu$ (resp. 4ee) channel. The advantage of tagging and measuring the intact proton in the final state is that it is possible to match the $\xi$ value, the proton momentum loss, as measured using either the dilepton information in the CMS central detector or directly the information from the proton tagged in the roman pots detector. 12 (resp. 8) events corresponding to a $<2 \sigma$ matching in the $\mu \mu$ (resp. ee) channels were observed. The significance is more than $5 \sigma$ for observing 20 events for a background of 3.85 $(1.49 \pm 0.07$ (stat) \pm 0.53 (syst) for $\mu \mu$ and $2.36 \pm 0.09$ (stat) \pm 0.47 (syst) for ee) [7]. The highest 
mass event was found to be $917 \mathrm{GeV}$. The ATLAS collaborations also observed the quasi-exclusive production of dileptons ( $e$ and $\mu$ ) using $14.6 \mathrm{fb}^{-1}$ at $13 \mathrm{TeV} .57$ (123) events are observed in $e e$ $(\mu \mu)$ channels leading to an evidence of more than $5 \sigma$, and the cross section was measured to be $\sigma_{e e+p}=11.0 \pm 2.6$ (stat) \pm 1.2 (syst) \pm 0.3 (lumi) fb and $\sigma_{\mu \mu+p}=7.2 \pm 1.6$ (stat) \pm 0.9 (syst) \pm 0.2 (lumi) fb [8].

\section{Light-by-light scattering}

Light-by-light scattering or the exclusive production of two photons was also observed by the CMS and ATLAS collaborations in $\mathrm{PbPb}$ collisions. The CMS collaborations look for two $\gamma$ back-to-back, and the main background is due to electrons misreconstructed as $\gamma$ and the QCD production of two photons, $g g \rightarrow \gamma \gamma$. The CMS selection was to request $E_{T_{\gamma}}>3 \mathrm{GeV},|\eta|<2.4$, $m_{\gamma \gamma}>5 \mathrm{GeV}, p_{T, \gamma \gamma}<1 \mathrm{GeV}$, and the acoplanarity to be less than 0.01 . 14 candidates were found with an estimated background of 4.0 \pm 0.1 [9]. We show the acoplanarity distribution in Fig. 3, left, showing a clear excess at low values corresponding to exclusive $\gamma \gamma$ production.

The ATLAS collaboration also measured a similar signal using 2015 data with the selection: $E_{T_{\gamma}}>3 \mathrm{GeV},|\eta|<2.37, m_{\gamma \gamma}>6 \mathrm{GeV}, p_{T, \gamma \gamma}<2 \mathrm{GeV}$, acoplanarity less than 0.01 , that led to the observation of 13 candidates, with an estimated background: $2.6 \pm 0.7$ [10]. The ATLAS collaboration redid this analysis with more luminosity using 2018 data with similar selection that led to 59 events with an expected background of $12 \pm 3$, and thus a $8.2 \sigma$ observation of light-by-light scattering [11]. We show the diphoton mass distribution in Fig. 3, right, that shows a clear excess with respect to backgrounds that is due to exclusive diphoton production.
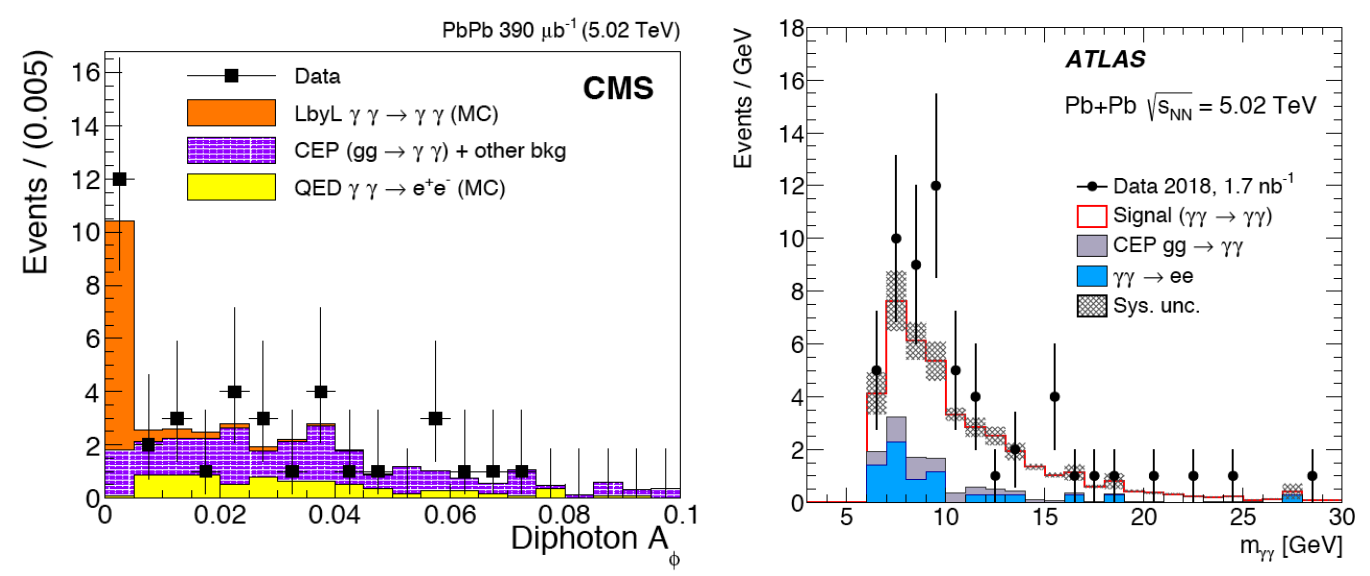

Figure 3: Observation of light-by-light scattering in $\mathrm{Pb} \mathrm{Pb}$ collisions by the CMS (left) and ATLAS (right) collaborations. The diphoton acoplanarity is shown on the left and the diphoton mass on the right.

The CMS collaboration looked for the first time for the exclusive production of two photons at high mass and two intact protons in the final state $p p \rightarrow p \gamma \gamma p$. This process (as well the exclusive production of $W W, Z Z, \gamma Z, t \bar{t}$ ) leads to possible high sensitivities to extra-dimensions, composite Higgs models, axion-like particles usually better by two of three orders of magnitude than more usual methods at the LHC. Anomalous couplings can appear via loops of new particles coupling 
to photons or via resonances decaying into two photons $[12,13,14]$. The advantage of tagging both protons is to obtain a negligible background after matching mass and rapidity of the photon and proton systems. The CMS collaboration searched for two isolated $\gamma$ in CMS, back-to-back, and 2 intact protons in PPS (matching in mass and rapidity the diphoton) with the requirements $M_{\gamma \gamma}>350 \mathrm{GeV}, p_{T, \gamma}>75 \mathrm{GeV}$, acoplanarity $<0.005$. The mass distribution is shown in Fig. 4, left, and shows a good agreement between data and the different backgrounds. Results are compatible with background hypothesis and lead to the first limits on photon quartic anomalous couplings $\left|\zeta_{1}\right|<3.710^{-13} \mathrm{GeV}^{-4}$ and $\left|\zeta_{2}\right|<7.710^{-13} \mathrm{GeV}^{-4}$ as shown in Fig. 4, right [15]. The study will be extended by ATLAS and CMS-TOTEM collaborations using the full accumulated luminosity to date of more than $100 \mathrm{fb}^{-1}$.

In this short report, we discussed the mots recent results from CMS and ATLAS concerning photon-induced processes, namely the first observation of $W W$ exclusive production, the observation of exclusive deletions in $\mathrm{PbPb}$ collisions, the observation of quasi-exclusive dilepton production in $p p$ collisions and finally the observation of light-by-light scattering in $P b P b$ collisions. Using the PPS proton detectors, the CMS and TOTEM collaborations also looked for diphoton production at high mass that led to the first limits on photon quartic anomalous couplings.
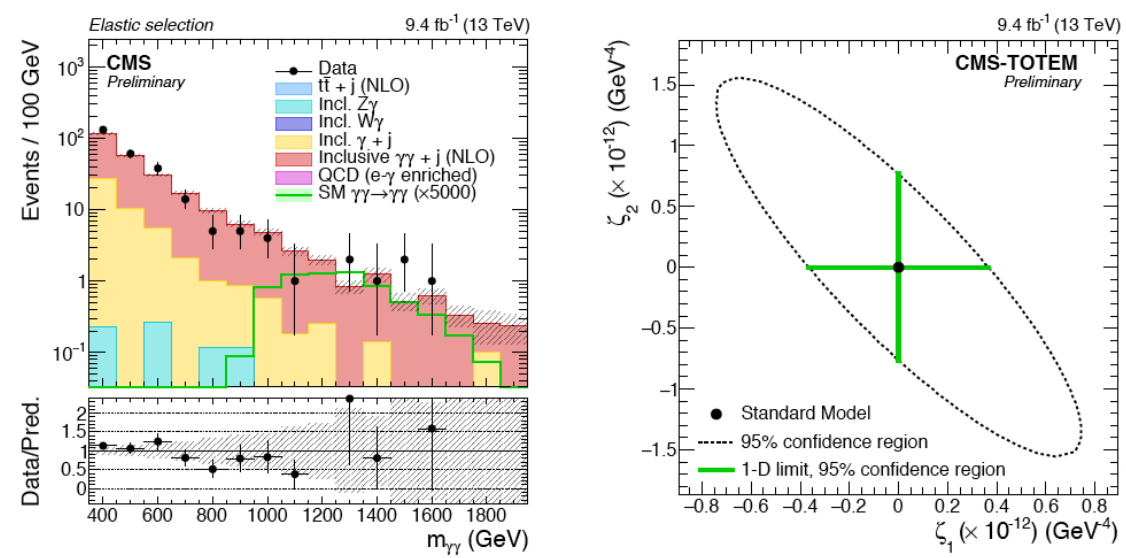

Figure 4: Search for high mass exclusive diphoton production with tagged protons in PPS in CMS. The diphoton mass distribution is shown on the left and is found to be in good agreement with Standard Model Monte Carlo expectations. The first exclusion contour in photon quartic anomalous couplings $\zeta_{1}$ and $\zeta_{2}$ is shown on the right.

\section{References}

[1] ATLAS Coll., Observation of photon-induced $W^{+} W^{\text {? }}$ production in $p p$ collisions at $\sqrt{s}=13 \mathrm{TeV}$ using the ATLAS detector, Phys. Lett. B816 (2021) 136.

[2] S.R. Klein, J. Nystrand, J. Seger, Y. Gorbunov, J. Butterworth, STARlight: A Monte Carlo simulation program for ultra-peripheral collisions of relativistic ions, Comput. Phys. Commun. 212 (2017) 258.

[3] CMS Coll., Observation of forward neutron multiplicity dependence of dimuon acoplanarity in ultraperipheral $\mathrm{PbPb}$ collisions at $\sqrt{s_{\mathrm{NN}}}=5.02 \mathrm{TeV}$, preprint ArXiv:2011.05239. 
[4] T.Sjöstrand et al.,An Introduction to PYTHIA 8.2, Comput. Phys. Commun. 191 (2015) 159.

[5] J.A.M. Vermaseren, Two Photon Processes at Very High-Energies, Nucl. Phys. B 229 (1983) 347.

[6] ATLAS Coll., Exclusive dimuon production in ultraperipheral $\mathrm{PbPb}$ collisions at $\sqrt{s}=5.02 \mathrm{TeV}$ with ATLAS, preprint ArXiv:2011.12211.

[7] CMS Coll., Observation of proton-tagged, central (semi)exclusive production of high-mass lepton pairs in pp collisions at $13 \mathrm{TeV}$ with the CMS-TOTEM precision proton spectrometer, JHEP 1807 (2018) 153.

[8] ATLAS Coll., Observation and Measurement of Forward Proton Scattering in Association with Lepton Pairs Produced via the Photon Fusion Mechanism at ATLAS, Phys. Rev. Lett. 125 (2020) 261801.

[9] CMS Coll., Evidence for light-by-light scattering and searches for axion-like particles in ultraperipheral $\mathrm{PbPb}$ collisions at $\sqrt{s}=5.02 \mathrm{TeV}$, Phys. Lett. B 797 (2019) 134826.

[10] ATLAS Coll., Evidence for light-by-light scattering in heavy-ion collisions with the ATLAS detector at the LHC, Nature physics 13 (2017) 852.

[11] ATLAS Coll., Observation of Light-by-Light Scattering in Ultraperipheral PbPb Collisions with the ATLAS Detector, Phys. Rev. Lett. 123 (2019) 052001.

[12] S. Fichet, G. von Gersdorff, B. Lenzi, C. Royon, M. Saimpert, Light-by-light scattering with intact protons at the LHC: from Standard Model to New Physics, JHEP 1502 (2015) 165; S. Fichet, G. von Gersdorff, O. Kepka, B. Lenzi, C. Royon, M. Saimpert, Probing new physics in diphoton production with proton tagging at the Large Hadron Collider, Phys.Rev. D89 (2014) 114004; S. Fichet, G. von Gersdorff, C. Royon, Measuring the Diphoton Coupling of a $750 \mathrm{GeV}$ Resonance, Phys. Rev. Lett. 116 (2016) no.23, 231801; S. Fichet, G. von Gersdorff, C. Royon, Scattering light by light at $750 \mathrm{GeV}$ at the LHC, Phys.Rev. D93 (2016) no.7, 075031

[13] C. Baldenegro, S. Fichet, G. von Gersdorff, C. Royon, Searching for axion-like particles with proton tagging at the LHC, JHEP 1806 (2018) 131; C. Baldenegro, S. Hassani, C. Royon, L. Schoeffel, Phys. Lett. B795 (2019) 339.

[14] C. Baldenegro, S. Fichet, G. von Gersdorff, C. Royon, Probing the anomalous $\gamma \gamma \gamma Z$ coupling at the LHC with proton tagging, JHEP 1706 (2017) 142; E. Chapon, C. Royon, O. Kepka, Anomalous quartic $W W \gamma \gamma, Z Z \gamma \gamma$, and trilinear $W W \gamma$ couplings in two-photon processes at high luminosity at the LHC, Phys.Rev. D81 (2010) 074003; O. Kepka , C. Royon, Anomalous $W W \gamma$ coupling in photon-induced processes using forward detectors at the LHC, Phys.Rev. D78 (2008) 073005

[15] CMS Coll., First search for exclusive diphoton production at high mass with tagged protons in proton-proton collisions at $\sqrt{s}=13 \mathrm{TeV}$, PAS EXO-18-014 